Article

\title{
Joint Optimization of Pico-Base-Station Density and Transmit Power for an Energy-Efficient Heterogeneous Cellular Network
}

\author{
Jie Yang ${ }^{1, *}$, Ziyu Pan ${ }^{1}$, Hengfei $\mathrm{Xu}^{1}$ and Han $\mathrm{Hu}^{2}$ \\ 1 Department of Communication Engineering, Nanjing Institute of Technology, Nanjing 211167, China; \\ panziyu@njit.edu.cn (Z.P.); xhf201@163.com (H.X.) \\ 2 Jiangsu Key Laboratory of Wireless Communications, Nanjing University of Post \& Telecommunications, \\ Nanjing 210023, China; han_h@njupt.edu.cn \\ * Correspondence: yangjie@njit.edu.cn
}

Received: 28 July 2019; Accepted: 25 September 2019; Published: 27 September 2019

\begin{abstract}
Heterogeneous cellular networks (HCNs) have emerged as the primary solution for explosive data traffic. However, an increase in the number of base stations (BSs) inevitably leads to an increase in energy consumption. Energy efficiency (EE) has become a focal point in HCNs. In this paper, we apply tools from stochastic geometry to investigate and optimize the energy efficiency (EE) for a two-tier HCN. The average achievable transmission rate and the total power consumption of all the BSs in a two-tier $\mathrm{HCN}$ is derived, and then the EE is formulated. In order to maximize EE, a one-dimensional optimization algorithm is used to optimize picocell BS density and transmit power. Based on this, an alternating optimization method aimed at maximizing EE is proposed to jointly optimize transmit power and density of picocell BSs. Simulation results validate the accuracy of the theoretical analysis and demonstrate that the proposed joint optimization method can obviously improve EE.
\end{abstract}

Keywords: energy efficiency; density; transmit power; optimization

\section{Introduction}

Mobile wireless communications have experienced explosive growth over the past decade, which has resulted in higher data rate and coverage requirements [1,2]. Heterogeneous cellular networks (HCNs) were introduced to address the exponential growth of mobile data traffic $[3,4]$. A typical multi-tier HCN consists of a macrocell for long-range coverage and several tiers of low-power, small cells for short-range coverage, such as picocells [5]. The deploying of multi-tier base stations (BSs) can enhance network throughput and mobile quality-of-service (QoS) [6,7]. Energy efficiency (EE) is one of the major parameters in the design of HCNs [8-12]. It has been revealed that $70 \%-80 \%$ of the energy consumption in a cellular network is attributed to the operations of the BSs [13]. Therefore, reducing energy consumption of BSs is critical to improve the EE of HCNs.

Stochastic geometry theory-Poisson point process (PPP) in particular-provides an effective and tractable method to analyze the performance of HCN [14-17]. Previous authors [18-21] have analyzed the total power consumption minimization frameworks under different performance constraints. In one study [22], the authors suggested that the power consumption of cellular networks be minimized by dynamically switching BSs on or off. In [23], the authors suggested power consumption be reduced via a joint sleeping strategy and power control. The works in [18-23] consider network power consumption rather than EE. In [24], the authors analyzed the EE of HCNs, however, no optimal EE scheme was given. In [25-27], the authors focused on the tradeoff between the spectral efficiency (SE) and the EE of HCNs, however, the closed-form expression of EE was not obtained. In [28], the authors analyzed the 
impact of BS transmit power on EE of HCNs and proposed an algorithm to find the optimal picocell BS transmit power in order to maximize EE. In [29], the EE was given in a tractable, closed-form formulation. Moreover, it has been mathematically proven that the EE is a unimodal and strictly pseudo-concave function in the transmit power given the density of the base stations, and likewise in the density of the base stations given the transmit power. In [30], the influence of parameters such as BSs' transmit powers and inter-site distances on EE was analyzed. In [31], the optimization of BS density to enhance EE through traffic-aware sleeping strategies in both one- and two-tier cellular networks was researched. In [32-36], area spectral efficiency (ASE) and EE of ultra-dense cellular networks were analyzed or optimized.

In this paper, we prefer to analyze and maximize the EE of a two-tier HCN. In previous works, EE has usually been defined as the ratio of sum rate to total power consumption [24,37-40]. In [24], the rate was computed based on the signal-to-interference ratio (SIR) threshold; the rate thus obtained should be the lower bound of the actual rate. The rate in [37-40] was based on either real-time signal to interference plus noise ratio (SINR) or SIR, which is equivalent to channel capacity. Unlike the previous works, in this paper, EE is defined as the ratio of average achievable transmission rate to BS power consumption. Considering that the service providers are interested in knowing the average rate they can provide to the users that are within coverage, the rate in this paper is computed on the condition that the user equipment (MU) is within coverage. In HCNs, the deployment of BSs has a notable impact on the EE [12,35]. In this paper, we intend to maximize the EE by jointly optimizing the deployment density and transmit power of picocell BSs.

The main contributions of this paper are as follows. First, we analyze and obtain the average achievable transmission rate of a two-tier HCN. Next, we investigate the total power consumption of all BSs in the per unit area of a two-tier HCN. Based on this, we derive the formulation of EE for a two-tier HCN with respect to MU density, BS densities, target signal-to-interference ratio (SIR), and power consumption of BSs. Then, we use a one-dimensional search algorithm to find the optimal density and transmit power, respectively. Finally, we propose a joint optimization strategy for picocell BS density and transmit power that can maximize EE.

The remainder of this paper is organized as follows. Section 2 introduces the system model. Section 3 analyzes the EE. The proposed EE optimization scheme is presented in Section 4. Simulation results are discussed in Section 5. Section 6 concludes the paper.

\section{System Model}

A downlink two-tier HCN comprising macrocells and picocells is considered. Macro BSs (MBSs) and pico-BSs (PBSs) are characterized by the corresponding density, transmit power, and target SIR. BSs in the $k$-th tier are modeled as a PPP $\phi_{k}(k=1,2)$ in the two-dimensional Euclidean plane, whose density, transmit power, and target SIR are denoted as $\lambda_{k}, P_{T k}$, and $\gamma_{k}$, respectively. Mobile user equipment (MUE) is modeled by another independent PPP with density $\lambda_{u}$. As shown in Figure 1, different cells in different tiers could be separate, overlapping, or included.

Given the stationary nature of the network model, network performance can be characterized by considering the throughput of a typical MUE located at the represented origin [11]. The typical MUE is called tagged MUE, and its serving BS is called a tagged BS. The distance between the tagged MUE and the tagged BS located at $x \in \phi_{k}(k=1,2)$ is denoted as $d_{x}$. The propagation channel is characterized by both path loss and small-scale fading. The received power at the tagged MUE attributed to the transmission from its serving BS is $P_{T k} h_{x} d_{x}^{-\alpha}$, where $\alpha>2$ is the path-loss exponent and $h_{x} \sim \exp (1)$ is a random variable modeling Rayleigh fading. All channel coefficients are assumed to be independent identically distributed (i.i.d). Thus, SINR of the tagged MUE is

$$
\operatorname{SINR}(x)=\frac{P_{T k} h_{x} d_{x}^{-\alpha}}{I_{x}+\sigma^{2}}=\frac{P_{T k} h_{x} d_{x}^{-\alpha}}{\sum_{k=1}^{2} \sum_{x^{\prime} \in \phi_{k} \backslash\{x\}} P_{T k} h_{x^{\prime}} d_{x^{\prime}}^{-\alpha}+\sigma^{2}}, x \in \phi_{k}
$$


where $I_{x}$ is the cumulative interference from all of the tiers when the tagged MUE is served by the tagged BS located at $x$, while $\sigma^{2}$ is the noise power. Typical HCNs are interference-limited; the noise power can be neglected in interference-limited cellular networks [14]. Therefore, in the following analysis, we use SIR instead of SINR. SIR of the tagged MUE can be written as

$$
\operatorname{SIR}(x)=\frac{P_{T k} h_{x} d_{x}^{-\alpha}}{I_{x}}=\frac{P_{T k} h_{x} d_{x}^{-\alpha}}{\sum_{k=1}^{2} \sum_{x^{\prime} \in \phi_{k} \backslash\{x\}} P_{T k} h_{x^{\prime}} d_{x^{\prime}}^{-\alpha}}, x \in \phi_{k}
$$

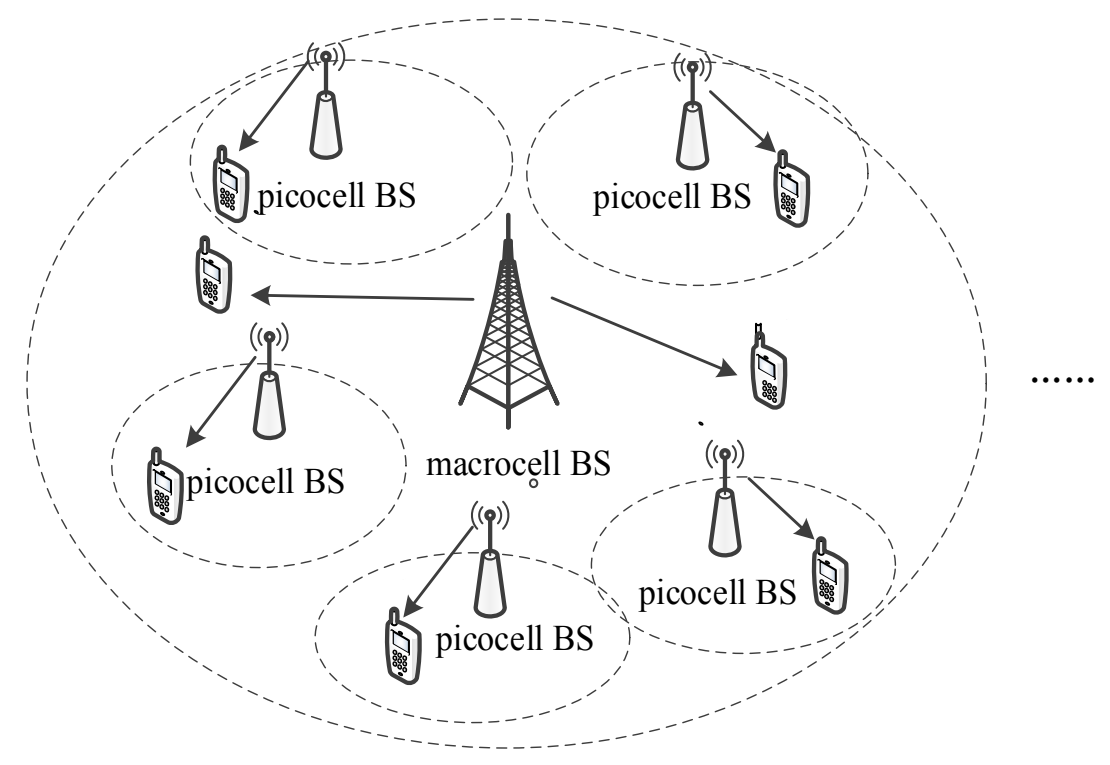

Figure 1. A two-tier, heterogeneous cellular network.

\section{Energy Efficiency Analysis}

Definition 1. In this paper, the energy efficiency is defined as the ratio of the average achievable rate to BS power consumption in the HCN. The EE indicates the total downlink rate that an HCN can achieve with certain power consumption.

$$
\eta_{E E}=\frac{R_{\text {total }}}{P_{\text {total }}}
$$

where $R_{\text {total }}$ is the total average achievable transmission rate and $P_{\text {total }}$ is the total power consumption of BSs. Firstly, we characterize the average achievable transmission rate. Then, we obtain the total power consumption of BSs and formulate the network EE.

\subsection{The Average Achievable Transmission Rate of A Two-Tier HCN}

We assume am open access strategy, where a typical MU can connect to a BS in any tier without any restriction (i.e., each MUE is served by the BS providing the highest received SIR).

Definition 2. The coverage probability of the target MUE served by the $k$-th tier BS is defined as

$$
P_{\operatorname{cov}}\left(\gamma_{k}\right)=\mathbb{P}\left(\cup_{x \in \phi_{k}} \operatorname{SIR}(x)>\gamma_{k}\right)=\mathbb{E}\left[1\left(\cup_{x \in \phi_{k}} \operatorname{SIR}(x)>\gamma_{k}\right)\right], k=1,2
$$


This definition represents the probability that the tagged MUE can achieve a target SIR $\gamma_{k}$ when it is served by a BS in the $k$-th tier. Assuming $\gamma_{k}>1(0 \mathrm{~dB})$, at most one BS in the entire network can provide SIR greater than the required threshold, $\gamma_{k}$ [14]. This assumption is easy to satisfy, except for with the users at the edge of the cell; the simulated and analytical results match reasonably well for a distinct minority (cell edge users). Under this assumption, Equation (4) can be written as [14]

$$
P_{\operatorname{cov}}\left(\gamma_{k}\right)=T(\alpha) \frac{\lambda_{k} P_{T k}^{2 / \alpha} \gamma_{k}{ }^{2 / \alpha}}{\sum_{k=1}^{2} \lambda_{k} P_{T k}{ }^{2 / \alpha}}, \gamma_{k}>1
$$

where $T(\alpha)=\frac{\sin (2 \pi / \alpha)}{2 \pi / \alpha}$. Under unit bandwidth, the average achievable rate $\mathrm{R}$ achieved by a randomly located MUE when it is within coverage can be expressed as [14]

$$
\begin{aligned}
\bar{R}= & \mathbb{E}\left[\left.\log \left(1+\max _{x \in \phi_{k},(k=1,2)} \operatorname{SIR}(x)\right)\right|_{x \in \phi_{k},(k=1,2)} \operatorname{SIR}(x)>\gamma_{k}\right] \\
& =\log \left(1+\gamma_{\min }\right)+\frac{\sum_{k=1}^{2} \int_{\gamma_{\min }}^{\infty} \frac{\max \left(\gamma_{k}, t\right)^{-2 / \alpha}}{1+t} d t P_{T k^{2 / \alpha}} \lambda_{k}}{\sum_{k=1}^{2} P_{T k} P^{2 / \alpha} \gamma_{k}^{-2 / \alpha} \lambda_{k}}
\end{aligned}
$$

where $\gamma_{\min }=\min _{k}\left\{\gamma_{k}\right\}$. We observe that the average rate expression involves only a single integral, which can be easily evaluated numerically. For the sake of tractability, we assume that each BS equally allocates the frequency resource among its serviced MUEs, and the bandwidth of the single frequency band is B. The average achievable rate of a randomly chosen MUE when it is within coverage of BSs in the $k$-th tier can be expressed as

$$
R_{k}=\frac{B}{U_{k}} \rho_{k} \bar{R}
$$

where $U_{k}$ is the average number of MUEs served by the tagged BS in the $k$-th tier, and $\rho_{k}$ is the average fraction of MUEs to be served by the $k$-th tier BSs. In other words, $\rho_{k}$ represents the proportion of the MUE coverage contributed by the BS in the $k$-th tier. As MUEs have different bandwidths when they access BSs in different tier, we need to distinguish which tier of BSs covers the MUEs. So, we use $\rho_{k}$ in Equation (7). Based on corollary 2 in [14], in open access mode, the average fraction of MUEs served by $k$-th tier BSs is

$$
\rho_{k}=\frac{\lambda_{k} P_{T k}^{2 / \alpha} \gamma_{k}^{-2 / \alpha}}{\sum_{k=1}^{2} \lambda_{k} P_{T k}^{2 / \alpha} \gamma_{k}^{-2 / \alpha}}
$$

According to Lemma 1 in [39] and Equation (9) in [24], $U_{k}$ can be expressed as

$$
U_{k}=1+1.28 \rho_{k} \lambda_{u} / \lambda_{k}
$$

According to the above analyses, the total average achievable rate can be readily defined.

Definition 3. The total average achievable rate of a two-tier HCN is defined as

$$
R_{\text {total }}=A \lambda_{u} R_{1}+A \lambda_{u} R_{2}
$$

where $A$ is the area of HCNs and $\lambda_{u}$ is the density of MUE. $R_{1}$ and $R_{2}$ represent the average achievable rate of a randomly chosen MUE when it is under coverage of BSs in the 1-th tier or the 2-th tier, respectively. Thus, we can get the total average achievable rate of a two-tier HCN.

\subsection{Total Power Consumption of Two-Tier HCN}

When referring to BS power consumption, it is generally agreed that BSs have two types of power consumption: circuit power consumption and transmit power consumption $[38,40]$. The circuit power 
consumption is caused by signal processing, site cooling, and battery backup. In this paper, we use the linear approximation model given by [41]:

$$
P_{k}=N_{T R k} \cdot P_{C k}+\theta_{k} P_{T k}
$$

where $N_{T R k}$ is the number of transceivers of a BS in the $k$-th tier; each transceiver serves one transmit antenna element. Here, $P_{C k}$ is the static power expenditure and $\theta_{k}$ is the slope of load-dependent power consumption. Thus, the total power consumption of all BSs in a two-tier HCN can be written as

$$
P_{\text {sum }}=A \lambda_{1}\left(N_{T R 1} \cdot P_{C 1}+\theta_{1} P_{T 1}\right)+A \lambda_{2}\left(N_{T R 2} \cdot P_{C 2}+\theta_{2} P_{T 2}\right)
$$

\subsection{Energy Efficiency of Two-Tier HCN}

Substituting Equations (10) and (12) into Equation (3), the energy efficiency of a two-tier HCN can be expressed as

$$
\eta_{E E}=\frac{A \lambda_{u} R_{1}+A \lambda_{u} R_{2}}{A \lambda_{1}\left(N_{T R 1} \cdot P_{C 1}+\theta_{1} P_{T 1}\right)+A \lambda_{2}\left(N_{T R 2} \cdot P_{C 2}+\theta_{2} P_{T 2}\right)}=\frac{B \lambda_{u} \bar{R}\left(\sum_{k=1}^{2} \frac{\rho_{k} \lambda_{k}}{\lambda_{k}+1.28 p_{k} \lambda_{u}}\right)}{\lambda_{1}\left(N_{T R 1} \cdot P_{C 1}+\theta_{1} P_{T 1}\right)+\lambda_{2}\left(N_{T R 2} \cdot P_{C 2}+\theta_{2} P_{T 2}\right)}
$$

where $\eta_{E E}$ represents network EE. Actually, $\gamma_{k}, N_{T R k}, \theta_{k}$, and $P_{C k}$ can be regarded as constants. From Equation (13), we can see that the network EE is only determined by the density of MU, the densities of BSs, and the transmit powers of BSs.

\section{Energy Efficiency Optimization}

Generally speaking, macrocell BSs are used to provide basic coverage, so their transmit power and density settings must ensure basic coverage. At the same time, due to the mobility and randomness of MUEs, their density cannot be controlled. Hence, we intend to improve the energy efficiency by jointly optimizing the transmit power and density of picocell BSs. Based on the above considerations, in this section, the density and the transmit powers of macrocell BSs and the density of MUE (i.e., $\lambda_{1}, P_{T 1}$, and $\lambda_{u}$ ) are assumed to be given. It is difficult to directly optimize the density and transmit power of picocell BSs jointly, so we first discuss the optimization based on density and power separately.

\subsection{Density Optimization Given the Transmit Power of Picocell BSs}

In this section, we analyze the optimal density of picocell BSs in order to maximize the EE formulated in Equation (13). The optimization problem can be expressed as

$$
\begin{gathered}
\max _{\lambda_{2}} \eta_{E E} \\
\text { s.t. } \lambda_{2} \geq \lambda_{\min }, \lambda_{2} \leq \lambda_{\max }
\end{gathered}
$$

In Equation (14), $\eta_{E E}$ is the objective function of the optimization problem, and density is the optimal variable. Here, $\lambda_{\min }>0$ and $\lambda_{\max }>0$ are the minimum and maximum density of the BSs, respectively. We assume, without loss of generality, $\lambda_{\min } \rightarrow \lambda_{1}$ and $\lambda_{\max } \rightarrow \lambda_{u}$. Through symbolic computation and simulation, we find that the objective function is a convex function and has the global optimum. According to the properties of convex functions, any extreme point of a convex function within a convex set is also its best point. Therefore, we use the one-dimensional optimization algorithm to find the optimal point of the objective function in the effective interval. In this paper, the golden section method is adopted to find the optimal solution of the optimization problem in Equation (14), and the solution is given by

$$
\widetilde{\lambda_{2}^{\text {opt }}}=\max \left\{\lambda_{\min }, \min \left\{\lambda_{2}^{*}, \lambda_{\max }\right\}\right\}
$$

where $\lambda_{2}^{*}$ is the only stationary point of the objective function. 


\subsection{Transmit Power Optimization Given the Density of Picocell BSs}

In this section, we analyze the optimal transmit power of picocell BSs. The optimization problem can be expressed as

$$
\begin{aligned}
& \max _{P_{T 2}} \eta_{E E} \\
& \text { s.t. } P_{T 2} \geq P_{\min }, P_{T 2} \leq P_{\max }
\end{aligned}
$$

In Equation (14), $\eta_{E E}$ is the objective function of the optimization problem, and transmit power is the optimal variable. Here, $P_{\min }>0$ and $P_{\max }>0$ are the minimum and maximum transmit power of the BSs, respectively. We assume, without loss of generality, that $P_{\min } \rightarrow 0$ and $P_{\max } \rightarrow P_{T 1}$. Through symbolic computation and simulation, we find that the objective function is also a convex function and has the global optimum. Similar to the solution to the optimization problem given in Equation (14), the golden section method is adopted to find the optimal solution to the optimization problem, and the solution is given by

$$
\widetilde{P_{T 2}^{o p t}}=\max \left\{P_{\min }, \min \left\{P_{\mathrm{T} 2}^{*}, P_{\max }\right\}\right\}
$$

where $P_{T 2}^{*}$ is the only stationary point of the objective function.

\subsection{Joint Optimization of Density and Transmit Power}

In Sections 4.1 and 4.2, we have solved the optimization problem formulated in Equation (14) with respect to $\lambda_{2}$ for a given $P_{T 2}$ and the optimization problem formulated in Equation (16) with respect to $P_{T 2}$ for a given $\lambda_{2}$, respectively. In this section, we tend to find the optimal pair $\left(P_{T 2}^{\text {opt }}, \lambda_{2}^{\text {opt }}\right)$ that jointly maximizes the EE in Equation (13). The joint optimization problem can be formulated as

$$
\begin{aligned}
& \max _{\lambda_{2}, P_{T 2}} \eta_{E E} \\
& \text { s.t. } P_{\min } \leq P_{T 2} \leq P_{\max }, \lambda_{\min } \leq \lambda_{2} \leq \lambda_{\max }
\end{aligned}
$$

By using these results obtained in Sections 4.1 and 4.2, we propose an alternating optimization method to solve the joint optimization problem given in Equation (18). This method iteratively optimizes $\lambda_{2}$ for a given $P_{T 2}$ and $P_{T 2}$ for a given $\lambda_{2}$ until convergence of the EE within a desired level of accuracy. The algorithm that solves Equation (18) based on the alternating optimization method is given in Algorithm 1.

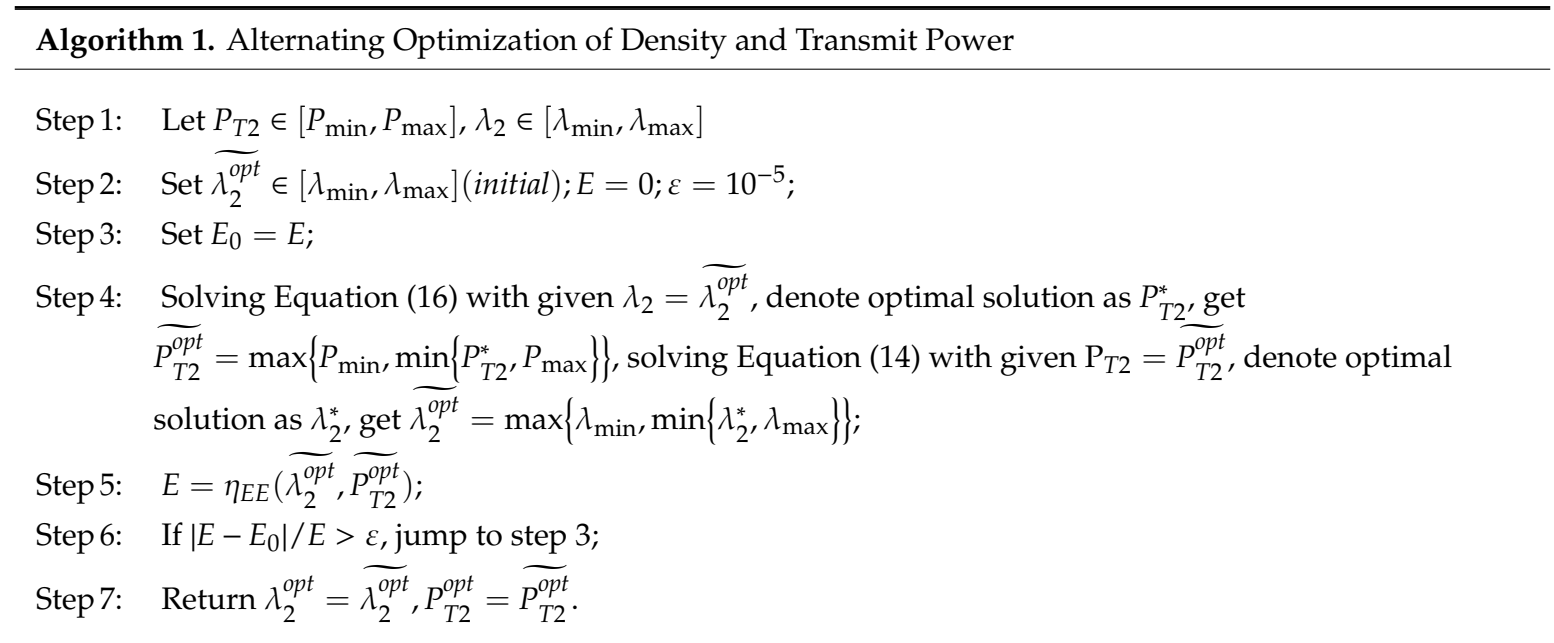

First, the optimization range of variables is given as $P_{T 2} \in\left[P_{\min }, P_{\max }\right], \lambda_{2} \in\left[\lambda_{\min }, \lambda_{\max }\right]$. Second, the initial iteration value of density is given as $\lambda_{2}^{\text {opt }} \in\left[\lambda_{\min }, \lambda_{\max }\right]$, the initial iteration value of EE is given as $E=0$, and the desired level of accuracy is given as $\varepsilon=10^{-5}$. Third, we iteratively optimize $\lambda_{2}$ for a given $P_{T 2}$ and $P_{T 2}$ for a given $\lambda_{2}$ until convergence of the EE within a desired level of accuracy. 
Last, the optimal solution pair and optimal pair $\left(P_{T 2}^{\text {opt }}, \lambda_{2}^{\text {opt }}\right)$ meeting the desired accuracy requirement is obtained.

\section{Simulation Results}

In this section, a series of numerical simulations are carried out to verify the accuracy of our derived EE equation and the effectiveness of our proposed optimization algorithm. Simulation parameters are listed in Table 1. For the power consumption model, we refer to the statistics in [41].

Table 1. Simulation parameters.

\begin{tabular}{cc}
\hline Parameters Name & Values \\
\hline Bandwidth & $10^{7} \mathrm{~Hz}$ \\
HetNet area & $10 \times 10 \mathrm{~km}^{2}$ \\
Density of MUEs, $\lambda_{\mathrm{u}}$ & $0.025 \mathrm{~m}^{-2}$ \\
Density of macrocell BS, $\lambda_{1}$ & $10^{-5} \mathrm{~m}^{-2}$ \\
Path-loss exponent, $\alpha$ & $\alpha=4$ \\
Circuit power consumption of MBS, $\mathrm{P}_{\mathrm{C} 1}$ & $130 \mathrm{~W}$ \\
Transmit power of MBS, $\mathrm{P}_{\mathrm{T} 1}$ & $20 \mathrm{~W}$ \\
Number of transceivers of MBS, $\mathrm{N}_{\mathrm{TR} 1}$ & 6 \\
Slope of MBS power consumption, $\theta_{1}$ & 4.7 \\
Circuit power consumption of PBS, $\mathrm{P}_{\mathrm{C} 2}$ & $6.8 \mathrm{~W}$ \\
Transmit power of PBS, $\mathrm{P}_{\mathrm{T} 2}$ & $0.13 \mathrm{~W}$ \\
Number of transceivers of PBS, $\mathrm{N}_{\mathrm{TR} 2}$ & 2 \\
Slope of PBS power consumption, $\theta_{2}$ & 4.0 \\
\hline
\end{tabular}

Figure 2 shows the impact of picocell BS density on EE. It can be seen that the simulation results are in line with the theoretical results, which validates the correctness of EE expression in Equation (13). We can see that if the MUE density, the macrocell BS density, and the power consumption of BS are given, there must exist an optimal picocell BS density that can maximize EE. It can be seen that the EE increases first and then decreases with the increase of the density of the picocell BS. The reason for the increase is that with the increase of the density of the picocell BS, since the distance from the picocell BS to the MUE is obviously smaller than that from the macrocell BS to MUE, more users will access the picocell BS. Additionally, the power consumption of the picocell BS is small, so EE will be improved at this time. However, when the density of the picocell BS is greater than a given value, the number of access users will be saturated, while a continuous increase of the density of the picocell BS will lead to an increase of power consumption, meaning the EE will gradually decrease.

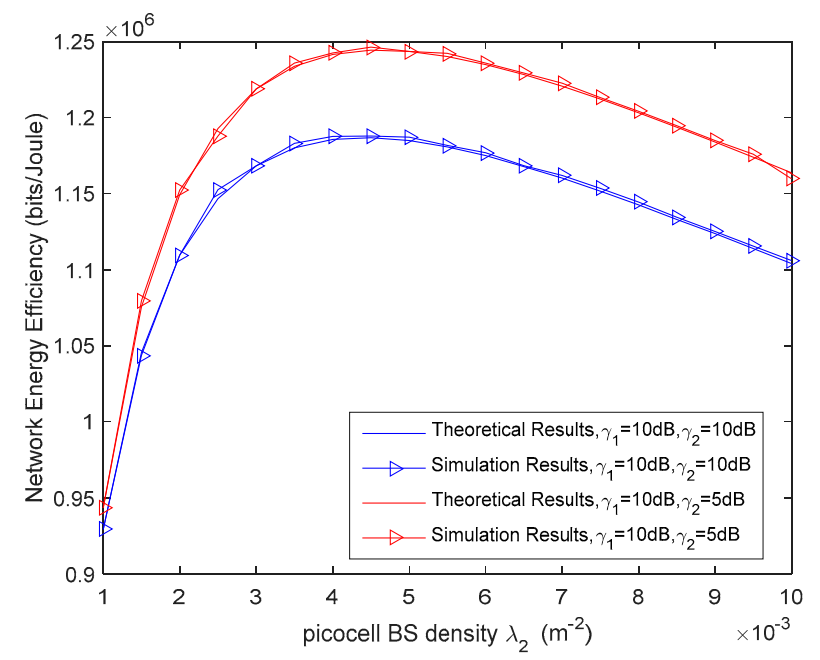

Figure 2. Network energy efficiency (EE) versus picocell base station (BS) density. 
In addition, both biased mode and unbiased mode are compared in this simulation. Unbiased mode means that the SIR threshold of a microcell BS is the same as that of a picocell BS, while biased mode means that the SIR threshold of a microcell BS is higher than that of a picocell BS. The target SIR for the picocell BSs is set to $10 \mathrm{~dB}$, while the target SIRs for picocell BSs are set to $10 \mathrm{~dB}$ in unbiased mode and $5 \mathrm{~dB}$ in biased mode, respectively. It can be seen that when $\lambda_{2}$ is not very low, bias technology helps to improve EE. This is because, in both unbiased mode and biased mode, more MUEs choose to access macrocell BSs when the distribution of picocell BSs is sparse, since macrocell BSs can provide higher SIR compared to picocell BSs. In this case, the EEs of the two modes are close to each other. With the increase of the picocell BSs density, in biased mode, increasingly more MUEs choose to access picocell BSs. Because the power consumption of a picocell BS is much lower than that of a macrocell BS, the EE of the biased mode is much higher.

Figure 3 gives the average EE against the transmit power of picocell BSs. Figure 3 shows the impact of picocell BS transmit power on EE. The target SIR for the macrocell BSs and picocell BSs are set to $10 \mathrm{~dB}$ and $5 \mathrm{~dB}$, respectively. It can be seen that the EE increases first and then decreases with the increase of the transmission power of the picocell BS. The reason for the increase is that with the increase of the transmission power of the picocell BS, since the SIR threshold of the micro-base station is low, increasingly more users will access the micro-base-station, while because the power of the picocell BS is small, EE will be improved at this time. However, when the transmission power of the picocell BS is greater than a given value, the number of access users will be saturated, and the continuous increase of the transmission power of the picocell BS will not lead to the increase of network throughput, so the EE will gradually decrease. It can also be seen that the simulation results are in line with the theoretical results. In addition, we can conclude that if $\lambda_{u}, \lambda_{1}, \lambda_{2}$, and $P_{T 1}$ are given, we can find an optimal $P_{T 2}$ that maximize EE.

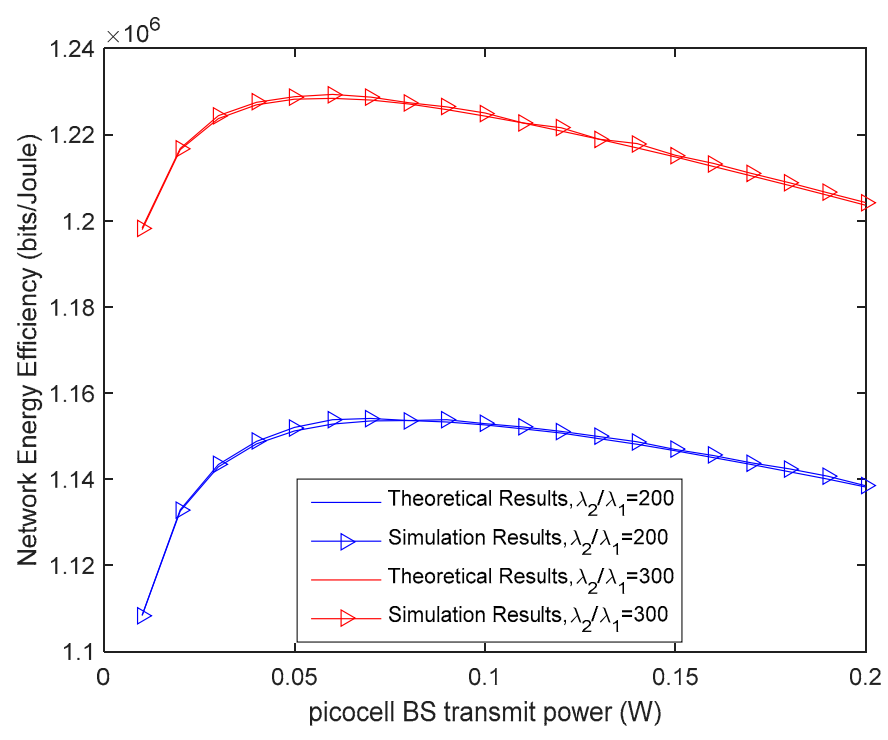

Figure 3. Network EE versus picocell BS transmit power.

In Figure 4, we evaluate the network EE with various target SIR thresholds to analyze the impact of different optimization schemes on EE. We use MATLAB (R2015b) to realize the golden section optimization algorithm. The CPU of our computer is $3.8 \mathrm{G}$, and the execution times to solve optimization Equations (14) and (16) are 0.0161 and 0.0159 s, respectively. The final optimal pair is obtained after 16 iterations of alternate optimization, and the execution time of our proposed optimization method is about $0.57 \mathrm{~s}$. 


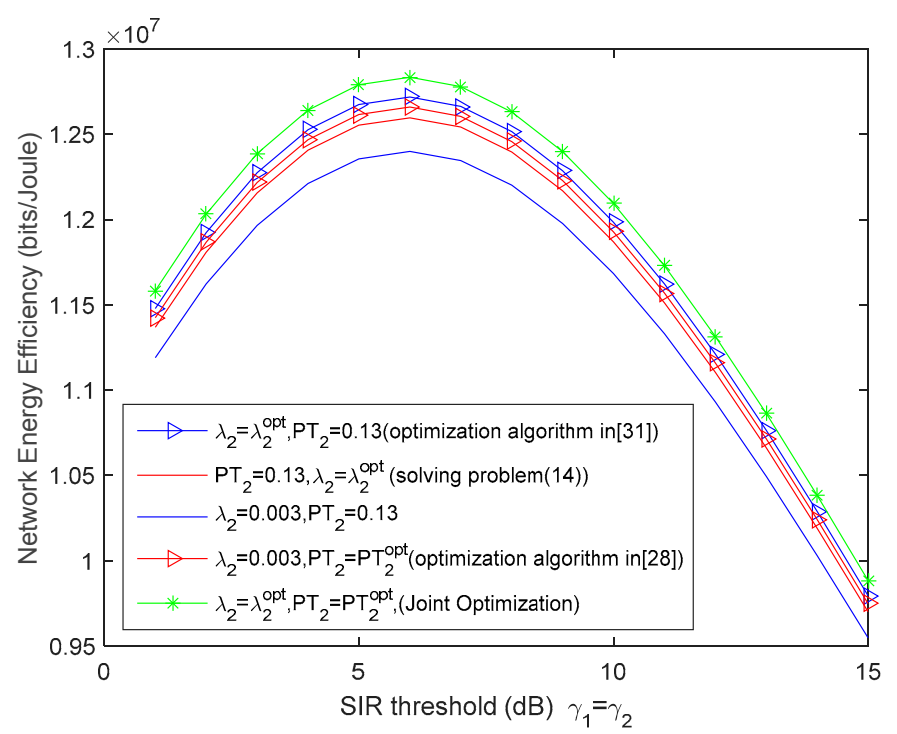

Figure 4. Network EE with fixed picocell BS density and optimal picocell BS density. Note: SIR = signal-to-interference ratio.

From Figure 4 we can see that with the increase of target SIR, the network EE curve rises first and then drops. This is because the achievable data rate of the tagged MUE increases as $\gamma_{1}$ grows, while the coverage probability decreases as target SIR increases. According to the optimization method given in Section 4.1, we calculate that $\lambda_{2}^{\text {opt }}=4.4 \times 10^{-3} \mathrm{~m}^{-2}$. It can be seen that the optimal solution $\lambda_{2}^{\text {opt }}$ is in agreement with the simulation results shown in Figure 2. According to the optimization method given in Section 4.2, which is also the optimization method proposed in [28], it can be calculated that $P_{T 2}^{o p t}=1.9 \times 10^{-2} \mathrm{~W}$. According to the proposed joint optimization algorithm, it can be calculated that the optimal pair is $\left(P_{T 2}^{\text {opt }}=2.9 \times 10^{-2} \mathrm{~W}, \lambda_{2}^{\text {opt }}=3.8 \times 10^{-3} \mathrm{~m}^{-2}\right)$. We also compare the proposed joint optimization scheme with the optimization algorithms proposed in [31], as well as the schemes for fixed power and density. The proposed joint optimization scheme can obviously improve network EE. Therefore, in HCNs, BS density and transmit power should be carefully designed. Otherwise, arbitrarily designed density or transmit power will decrease the network EE.

\section{Conclusions}

In this paper, we use stochastic geometry approach to determine the energy efficiency of picocell BS density and transmit power for a two-tier HCN. We formulate and derive the expression of the network EE in terms of the MUE density, BS density, target SIR, and the transmit powers of BSs. We propose an alternating optimization scheme to achieve joint power and density optimization. The scheme can achieve optimal pairing of transmit power and density. Simulation results validated the analysis and proved the effectiveness of the EE joint optimization scheme. We found that compared with unilateral optimization of density or transmit power, joint optimization can improve system EE more effectively. This work can offer theoretical references for the design and deployment of dense HCNs.

Author Contributions: Conceptualization, J.Y. and Z.P.; methodology, J.Y.; simulation, H.H. and J.Y.; formal analysis, J.Y. and H.X.; writing — original draft preparation, J.Y. and Z.P.; writing-review and editing, H.X.

Funding: This work was supported by the National Natural Science Foundation of China No. 61701221, 61901211, the Natural Science Foundation of Jiangsu Province (China) No. BK20160781, Jiangsu Higher Education Institutions Natural Science Foundation No. 18KJB510017, Nantong Science and Technology Project No. JC2018127, and Research Initiation Fund Project of Nanjing Institute of Technology No. YKJ201869.

Conflicts of Interest: The authors declare no conflict of interest. 


\section{References}

1. Andrews, J.G.; Buzzi, S.; Wan, C.; Hanly, S.V.; Lozano, A.; Soong, A.C.K.; Zhang, J.C. What Will 5G Be. IEEE J. Sel. Areas Commun. 2014, 32, 1065-1082. [CrossRef]

2. Kamel, M.; Hamouda, W.; Youssef, A. Ultra-Dense Networks: A Survey. IEEE Commun. Surv. Tutor. 2016, 18, 2522-2545. [CrossRef]

3. Yunas, S.F.; Valkama, M.; Niemelä, J. Spectral and energy efficiency of ultra-dense networks under different deployment strategies. IEEE Commun. Mag. 2015, 53, 90-100. [CrossRef]

4. Wang, H.; Leung, S.; Song, R. Uplink Area Spectral Efficiency Analysis for Multichannel Heterogeneous Cellular Networks with Interference Coordination. IEEE Access 2018, 6, 14485-14497. [CrossRef]

5. Saha, C.; Afshang, M.; Dhillon, H.S. Enriched K-Tier HCN Model to Enable the Analysis of User-Centric Small Cell Deployments. IEEE Trans. Wirel. Commun. 2016, 16, 2835-2849.

6. Mirahsan, M.; Schoenen, R.; Yanikomeroglu, H. HetHCNs: Heterogeneous Traffic Distribution in Heterogeneous Wireless Cellular Networks. IEEE J. Sel. Areas Commun. 2015, 33, 2252-2265. [CrossRef]

7. Elsawy, H.; Sultan-Salem, A.; Alouini, M.S.; Win, M.Z. Modeling and Analysis of Cellular Networks Using Stochastic Geometry: A Tutorial. IEEE Commun. Surv. Tutor. 2017, 19, 167-203. [CrossRef]

8. She, C.; Yang, C. Energy Efficiency and Delay in Wireless Systems: Is Their Relation Always a Tradeoff. IEEE Trans. Wirel. Commun. 2016, 15, 7215-7228. [CrossRef]

9. Chen, Z.; Qiu, L.; Liang, X. Area Spectral Efficiency Analysis and Energy Consumption Minimization in Multiantenna Poisson Distributed Networks. IEEE Trans. Wirel. Commun. 2016, 15, 4862-4874.

10. Logambigai, R.; Kannan, A. Energy conservation routing algorithm for wireless sensor networks using hybrid optimisation approach. Int. J. Commun. Netw. Distrib. Syst. 2018, 20, 352-371. [CrossRef]

11. Saravanan, R. Energy efficient QoS routing for mobile ad hoc networks. Int. J. Commun. Netw. Distrib. Syst. 2018, 20, 372-388. [CrossRef]

12. Su, L.; Yang, C.; Chih-Lin, I. Energy and Spectral Efficient FreqMUncy Reuse of Ultra Dense Networks. IEEE Trans. Wirel. Commun. 2016, 15, 5384-5398.

13. Thompson, J.; Ge, X.; Wu, H.C.; Lrmer, R.; Jiang, H.; Fettweis, G.; Alamouti, S. 5G Wireless Communication Systems: Prospects and Challenges Part 2. IEEE Commun. Mag. 2014, 52, 24-26. [CrossRef]

14. Dhillon, H.S.; Ganti, R.K.; Baccelli, F.; Andrews, J.G. Modeling and Analysis of K-Tier Downlink Heterogeneous Cellular Networks. IEEE J. Sel. Areas Commun. 2012, 30, 550-560. [CrossRef]

15. Jo, H.S.; Sang, Y.J.; Xia, P.; Andrews, J.G. Heterogeneous Cellular Networks with Flexible Cell Association: A Comprehensive Downlink SINR Analysis. IEEE Trans. Wirel. Commun. 2011, 11, 3484-3495. [CrossRef]

16. Renzo, M.D.; Guidotti, A.; Corazza, G.E. Average Rate of Downlink Heterogeneous Cellular Networks over Generalized Fading Channels: A Stochastic Geometry Approach. IEEE Trans. Commun. 2013, 61, 3050-3071. [CrossRef]

17. Subhankar, C.; Susmit, D. Poisson point process-based network modelling and performance analysis of multi-hop D2D chain relay formation in heterogeneous wireless network. Int. J. Commun. Netw. Distrib. Syst. 2019, 22, 98-122.

18. Xiang, L.; Ge, X.; Wang, C.X.; Li, F.Y.; Reichert, F. Energy Efficiency Evaluation of Cellular Networks Based on Spatial Distributions of Traffic Load and Power Consumption. IEEE Trans. Wirel. Commun. 2013, 12, 961-973. [CrossRef]

19. Cao, D.; Zhou, S.; Niu, Z. Optimal Combination of Base Station Densities for Energy-Efficient Two-Tier Heterogeneous Cellular Networks. IEEE Trans. Wirel. Commun. 2013, 12, 4350-4362. [CrossRef]

20. Peng, J.; Hong, P.; Xue, K. Energy-Aware Cellular Deployment Strategy under Coverage Performance Constraints. IEEE Trans. Wirel. Commun. 2015, 14, 69-80. [CrossRef]

21. Mugume, E.; So, D.K.C.; Alsusa, E. Energy Efficient Deployment of Dense Heterogeneous Cellular Networks. In Proceedings of the IEEE Global Communications Conference (GLOBECOM), San Diego, CA, USA, 6-10 December 2015; pp. 1-6.

22. Yu, N.; Miao, Y.; Mu, L.; Du, H.; Huang, H.; Jia, X. Minimizing Energy Cost by Dynamic Switching ON/OFF Base Stations in Cellular Networks. IEEE Trans. Wirel. Commun. 2016, 15, 7457-7469. [CrossRef]

23. Luo, J.; Chen, Q.; Tang, L. Reducing Power Consumption by Joint Sleeping Strategy and Power Control in Delay-Aware C-RAN. IEEE Access 2018, 6, 14655-14667. [CrossRef] 
24. Zhang, T.; Zhao, J.; An, L.; Liu, D. Energy Efficiency of Base Station Deployment in Ultra Dense HCNs: A Stochastic Geometry Analysis. IEEE Wirel. Commun. Lett. 2016, 5, 184-187. [CrossRef]

25. Coskun, C.C.; Ayanoglu, E. Energy- and Spectral-Efficient Resource Allocation Algorithm for Heterogeneous Networks. IEEE Trans. Veh. Technol. 2018, 67, 590-603. [CrossRef]

26. Aydin, O.; Jorswieck, E.A.; Aziz, D.; Zappone, A. Energy-Spectral Efficiency Trade-Offs in 5G Multi-Operator Networks with Heterogeneous Constraints. IEEE Trans. Wirel. Commun. 2017, 16, 5869-5881. [CrossRef]

27. Zhao, G.; Chen, S.; Zhao, L.; Hanzo, L. Energy-Spectral-Efficiency Analysis and Optimization of Heterogeneous Cellular Networks: A Large-Scale User-Behavior Perspective. IEEE Trans. Veh. Technol. 2018, 67, 4098-4112. [CrossRef]

28. Jie, Y.; Zhenjian, T.; Han, H.; Yonghong, C. Energy-efficient picocell base station power control in heterogeneous cellular network. In Proceedings of the IEEE 17th International Conference on Communication Technology (ICCT), Chengdu, China, 27-30 October 2017; pp. 553-557.

29. Di Renzo, M.; Zappone, A.; Lam, T.T.; Debbah, M. System-Level Modeling and Optimization of the Energy Efficiency in Cellular Networks-A Stochastic Geometry Framework. IEEE Trans. Wirel. Commun. 2018, 17, 2539-2556. [CrossRef]

30. Lorincz, J.; Matijevic, T. Energy-efficiency analyses of heterogeneous macro and micro base station sites. Comput. Electr. Eng. 2014, 40, 330-349. [CrossRef]

31. Li, L.; Peng, M.; Yang, C.; Wu, Y. Optimization of Base-Station Density for High Energy-Efficient Cellular Networks with Sleeping Strategies. IEEE Trans. Veh. Technol. 2015, 65, 7501-7514. [CrossRef]

32. Zhao, G.; Chen, S.; Qi, L.; Zhao, L.; Hanzo, L. Mobile-Traffic-Aware Offloading for Energy- and Spectral-Efficient Large-Scale D2D-Enabled Cellular Networks. IEEE Trans. Wirel. Commun. 2019, 18, 3251-3264. [CrossRef]

33. Luo, Y.; Shi, Z.; Bu, F.; Xiong, J. Joint Optimization of Area Spectral Efficiency and Energy Efficiency for Two-Tier Heterogeneous Ultra-Dense Networks. IEEE Access 2019, 7, 12073-12086. [CrossRef]

34. Krauss, R.; Brante, G.; Rayel, O.K.; Souza, R.D.; Onireti, O.; Imran, M.A. Energy Efficiency of Multiple Antenna Cellular Networks Considering a Realistic Power Consumption Model. IEEE Trans. Green Commun. Netw. 2019, 3, 1-10. [CrossRef]

35. Cai, Y.; Ni, Y.; Zhang, J.; Zhao, S.; Zhu, H. Energy efficiency and spectrum efficiency in underlay device-to-device communications enabled cellular networks. China Commun. 2019, 16, 16-34.

36. Mollahasani, S.; Onur, E. Density-Aware, Energy- and Spectrum-Efficient Small Cell Scheduling. IEEE Access 2019, 7, 65852-65869. [CrossRef]

37. Tang, J.; So, D.K.C.; Alsusa, E.; Hamdi, K.; Shojaeifard, A.; Wong, K. Energy Efficiency Optimization for Heterogeneous Cellular Networks. In Proceedings of the 2017 IEEE 85th Vehicular Technology Conference (VTC Spring), Sydney, Australia, 4-7 June 2017; pp. 1-6.

38. Sui, X.; Zhao, Z.; Li, R.; Zhang, H. Energy Efficiency Analysis of Heterogeneous Cellular Networks with Downlink and Uplink Decoupling. In Proceedings of the 2015 IEEE Global Communications Conference (GLOBECOM), San Diego, CA, USA, 6-10 December 2015; pp. 1-7.

39. Singh, S.; Dhillon, H.S.; Andrews, J.G. Offloading in Heterogeneous Networks: Modeling, Analysis, and Design Insights. IEEE Trans. Wirel. Commun. 2013, 12, 2484-2497. [CrossRef]

40. Lorincz, J.; Matijevic, T.; Petrovic, G. On interdependence among transmit and consumed power of macro base station technologies. Comput. Commun. 2014, 50, 10-28. [CrossRef]

41. Auer, G.; Giannini, V.; Desset, C.; Gódor, L.; Skillermark, P.; Olsson, M.; Imran, M.A.; Sabella, D.; Gonzalez, M.J.; Blume, O.; et al. How much energy is needed to run a wireless network? IEEE Wirel. Commun. 2011, 18, 40-49. [CrossRef]

(C) 2019 by the authors. Licensee MDPI, Basel, Switzerland. This article is an open access article distributed under the terms and conditions of the Creative Commons Attribution (CC BY) license (http://creativecommons.org/licenses/by/4.0/). 